\title{
RESTRICTED PERMUTATIONS, CONTINUED FRACTIONS, AND CHEBYSHEV POLYNOMIALS
}

\author{
TOufiK Mansour* $^{*}$ And Alek Vainshtein ${ }^{\dagger}$ \\ * Department of Mathematics \\ $\dagger$ Department of Mathematics and Department of Computer Science \\ University of Haifa, Haifa, Israel 31905 \\ tmansur@study.haifa.ac.il, alek@mathcs.haifa.ac.il
}

Submitted: December 21, 1999; Accepted: February 27, 2000.

\begin{abstract}
Let $f_{n}^{r}(k)$ be the number of 132-avoiding permutations on $n$ letters that contain exactly $r$ occurrences of $12 \ldots k$, and let $F_{r}(x ; k)$ and $F(x, y ; k)$ be the generating functions defined by $F_{r}(x ; k)=\sum_{n \geqslant 0} f_{n}^{r}(k) x^{n}$ and $F(x, y ; k)=\sum_{r \geqslant 0} F_{r}(x ; k) y^{r}$. We find an explicit expression for $F(x, y ; k)$ in the form of a continued fraction. This allows us to express $F_{r}(x ; k)$ for $1 \leqslant r \leqslant k$ via Chebyshev polynomials of the second kind.
\end{abstract}

2000 Mathematics Subject Classifichtion: Primary 05A05, 05A15; Secondary 30B70, 42C05 


\section{INTRODUCTION}

Let $[p]=\{1, \ldots, p\}$ denote a totally ordered alphabet on $p$ letters, and let $\alpha=$ $\left(\alpha_{1}, \ldots, \alpha_{m}\right) \in\left[p_{1}\right]^{m}, \beta=\left(\beta_{1}, \ldots, \beta_{m}\right) \in\left[p_{2}\right]^{m}$. We say that $\alpha$ is order-isomorphic to $\beta$ if for all $1 \leqslant i<j \leqslant m$ one has $\alpha_{i}<\alpha_{j}$ if and only if $\beta_{i}<\beta_{j}$. For two permutations $\pi \in \mathfrak{S}_{n}$ and $\tau \in \mathfrak{S}_{k}$, an occurrence of $\tau$ in $\pi$ is a subsequence $1 \leqslant i_{1}<i_{2}<\cdots<i_{k} \leqslant n$ such that $\left(\pi_{i_{1}}, \ldots, \pi_{i_{k}}\right)$ is order-isomorphic to $\tau$; in such a context $\tau$ is usually called the pattern. We say that $\pi$ avoids $\tau$, or is $\tau$-avoiding, if there is no occurrence of $\tau$ in $\pi$. The set of all $\tau$-avoiding permutations of all possible sizes including the empty permutation is denoted $\mathfrak{S}(\tau)$. Pattern avoidance proved to be a useful language in a variety of seemingly unrelated problems, from stack sorting [5] to singularities of Schubert varieties [6]. A complete study of pattern avoidance for the case $\tau \in \mathfrak{S}_{3}$ is carried out in [11]. For the case $\tau \in \mathfrak{S}_{4}$ see [14, $11,12,1]$.

A natural generalization of pattern avoidance is the restricted pattern inclusion, when a prescribed number of occurrences of $\tau$ in $\pi$ is required. Papers [8] and [3] contain simple expressions for the number of permutations containing exactly one 123 and 132 patterns, respectively. The main result of [B2] is that the generating function for the number of permutations containing exactly $r 132$ patterns is a rational function in variables $x$ and $\sqrt{1-4 x}$. This proves a particular case of the general conjecture of Noonan and Zeilberger [9] which is that for any set $T$ of patterns, the sequence of numbers enumerating permutations having a prescribed number of occurrences of patterns in $T$ is $P$-recursive. Recent paper [10] presents the generating function for the number of 132-avoiding permutations that contain a prescribed number of 123 patterns. The generating function is given in the form of a continued fraction. In the present note we generalize the argument of [10] to get the generating function for the number of 132-avoiding permutations that contain a prescribed number of $12 \ldots k$ patterns for arbitrary $k \geqslant 3$. The study of the obtained continued fraction allows us to recover and to generalize the result of [4] that relates the number of 132 -avoiding permutations that contain no $12 \ldots k$ patterns to Chebyshev polynomials of the second kind.

The authors are grateful to C. Krattenthaler, H. Wilf, and anonymous referee for useful comments concerning Theorems 4.1 and 4.2 .

\section{Continued Fractions}

Let $f_{n}^{r}(k)$ stand for the number of 132-avoiding permutations on $n$ letters that contain exactly $r$ occurrences of $12 \ldots k$. We denote by $F(x, y ; k)$ the generating function of the sequence $\left\{f_{n}^{r}(k\}\right)$, that is,

$$
F(x, y ; k)=\sum_{n \geqslant 0} \sum_{r \geqslant 0} f_{n}^{r}(k) x^{n} y^{r} .
$$

Our first result is a natural generalization of the main theorem of [10]. 
Theorem 2.1. The generating function $F(x, y ; k)$ for $k \geqslant 1$ is given by the continued fraction

$$
F(x, y ; k)=\frac{1}{1-\frac{x y^{d_{1}}}{1-\frac{x y^{d_{2}}}{1-\frac{x y^{d_{3}}}{\cdots}}},}
$$

where $d_{i}=\left(\begin{array}{l}i-1 \\ k-1\end{array}\right)$, and $\left(\begin{array}{l}a \\ b\end{array}\right)$ is assumed 0 whenever $a<b$ or $b<0$.

Proof. Following [10] we define $\eta_{j}(\pi), j \geqslant 1$, as the number of occurrences of $12 \ldots j$ in $\pi$. Define $\eta_{0}(\pi)=1$ for any $\pi$, which means that the empty pattern occurs exactly once in each permutation. The weight of a permutation $\pi$ is a monomial in $k$ independent variables $q_{1}, \ldots, q_{k}$ defined by

$$
w_{k}(\pi)=\prod_{j=1}^{k} q_{j}^{\eta_{j}(\pi)} .
$$

The total weight is a polynomial

$$
W_{k}\left(q_{1}, \ldots, q_{k}\right)=\sum_{\pi \in \mathfrak{S}(132)} w_{k}(\pi) .
$$

The following proposition is implied immediately by the definitions.

Proposition 2.1. $F(x, y ; k)=W_{k}(x, 1, \ldots, 1, y)$ for $k \geqslant 2$, and $F(x, y ; 1)=$ $W_{1}(x y)$.

We now find a recurrence relation for the numbers $\eta_{j}(\pi)$. Let $\pi \in \mathfrak{S}_{n}$, so that $\pi=\left(\pi^{\prime}, n, \pi^{\prime \prime}\right)$.

Proposition 2.2. For any $j \geqslant 1$ and any nonempty $\pi \in \mathfrak{S}(132)$

$$
\eta_{j}(\pi)=\eta_{j}\left(\pi^{\prime}\right)+\eta_{j}\left(\pi^{\prime \prime}\right)+\eta_{j-1}\left(\pi^{\prime}\right) .
$$

Proof. Let $l=\pi^{-1}(n)$. Since $\pi$ avoids 132, each number in $\pi^{\prime}$ is greater than any of the numbers in $\pi^{\prime \prime}$. Therefore, $\pi^{\prime}$ is a 132-avoiding permutation of the numbers $\{n-l+1, n-l+2, \ldots, n-1\}$, while $\pi^{\prime \prime}$ is a 132 -avoiding permutation of the numbers $\{1,2, \ldots, n-l\}$. On the other hand, if $\pi^{\prime}$ is an arbitrary 132-avoiding permutation of the numbers $\{n-l+1, n-l+2, \ldots, n-1\}$ and $\pi^{\prime \prime}$ is an arbitrary 132-avoiding permutation of the numbers $\{1,2, \ldots, n-l\}$, then $\pi=\left(\pi^{\prime}, n, \pi^{\prime \prime}\right)$ is 132-avoiding. Finally, if $\left(i_{1}, \ldots, i_{j}\right)$ is an occurrence of $12 \ldots j$ in $\pi$ then either $i_{j}<l$, and so it is also an occurrence of $12 \ldots j$ in $\pi^{\prime}$, or $i_{1}>l$, and so it is also an occurrence of $12 \ldots j$ in $\pi^{\prime \prime}$, or $i_{j}=l$, and so $\left(i_{1}, \ldots, i_{j-1}\right)$ is an occurrence of $12 \ldots j-1$ in $\pi^{\prime}$. The result follows. 
Now we are able to find the recurrence relation for the total weight $W$. Indeed, by Proposition 2.2,

$$
\begin{aligned}
W_{k}\left(q_{1}, \ldots, q_{k}\right) & =1+\sum_{\varnothing \neq \pi \in \mathfrak{S}(132)} \prod_{j=1}^{k} q_{j}^{\eta_{j}\left(\pi^{\prime}\right)+\eta_{j}\left(\pi^{\prime \prime}\right)+\eta_{j-1}\left(\pi^{\prime}\right)} \\
& =1+\sum_{\pi^{\prime} \in \mathfrak{S}(132)} \sum_{\pi^{\prime \prime} \in \mathfrak{S}(132)} \prod_{j=1}^{k} q_{j}^{\eta_{j}\left(\pi^{\prime \prime}\right)} \cdot q_{1} \prod_{j=1}^{k-1}\left(q_{j} q_{j+1}\right)^{\eta_{j}\left(\pi^{\prime}\right)} \cdot q_{k}^{\eta_{k}\left(\pi^{\prime}\right)} \\
& =1+q_{1} W_{k}\left(q_{1}, \ldots, q_{k}\right) W_{k}\left(q_{1} q_{2}, q_{2} q_{3}, \ldots, q_{k-1} q_{k}, q_{k}\right) .
\end{aligned}
$$

For any $d \geqslant 0$ and $1 \leqslant m \leqslant k$ define

$$
\mathbf{q}^{d, m}=\prod_{j=1}^{k} q_{j}^{\left(\begin{array}{c}
d \\
j-m
\end{array}\right)}
$$

recall that $\left(\begin{array}{l}a \\ b\end{array}\right)=0$ if $a<b$ or $b<0$. The following proposition is implied immediately by the well-known properties of binomial coefficients.

Proposition 2.3. For any $d \geqslant 0$ and $1 \leqslant m \leqslant k$

$$
\mathbf{q}^{d, m} \mathbf{q}^{d, m+1}=\mathbf{q}^{d+1, m} .
$$

Observe now that $W_{k}\left(q_{1}, \ldots, q_{k}\right)=W_{k}\left(\mathbf{q}^{0,1}, \ldots, \mathbf{q}^{0, k}\right)$ and that by (1) and Proposition 2.3

$$
W_{k}\left(\mathbf{q}^{d, 1}, \ldots, \mathbf{q}^{d, k}\right)=1+\mathbf{q}^{d, 1} W_{k}\left(\mathbf{q}^{d, 1}, \ldots, \mathbf{q}^{d, k}\right) W_{k}\left(\mathbf{q}^{d+1,1}, \ldots, \mathbf{q}^{d+1, k}\right),
$$

therefore

$$
W_{k}\left(q_{1}, \ldots, q_{k}\right)=\frac{1}{1-\frac{\mathbf{q}^{0,1}}{1-\frac{\mathbf{q}^{1,1}}{1-\frac{\mathbf{q}^{2,1}}{\ldots}}}} .
$$

To obtain the continued fraction representation for $F(x, y ; k)$ it is enough to use Proposition 2.1 and to observe that

$$
\left.\mathbf{q}^{d, 1}\right|_{q_{1}=x, q_{2}=\cdots=q_{k-1}=1, q_{k}=y}=x y^{\left(\begin{array}{c}
d \\
k-1
\end{array}\right) .}
$$

Remark. For $k=1$ one recovers from Theorem 2.1 the well-known generating function for the Catalan numbers, $(1-\sqrt{1-4 z}) / 2 z$. This result also follows immediately from Proposition 2.1 and equation (1), which for $k=1$ is reduced to $W_{1}(q)=1+q W_{1}^{2}(q)$. 


\section{Chebyshev polynomials}

Let us denote by $F_{r}(x ; k)$ the generating function of the sequence $\left\{f_{n}^{r}(k)\right\}$ for a given $r$, that is,

$$
F_{r}(x ; k)=\sum_{n \geqslant 0} f_{n}^{r}(k) x^{n}
$$

Recall that $F(x, y ; k)=\sum_{r \geqslant 0} F_{r}(x ; k) y^{r}$. In this section we find explicit expressions for $F_{r}(x ; k)$ in the case $0 \leqslant r \leqslant k$.

Consider a recurrence relation

$$
T_{j}=\frac{1}{1-x T_{j-1}}, \quad j \geqslant 1 .
$$

The solution of (2) with the initial condition $T_{0}=0$ is denoted by $R_{j}(x)$, and the solution of (2) with the initial condition

$$
T_{0}=G(x, y ; k)=\frac{y}{1-\frac{x y^{\left(\begin{array}{l}
k \\
1
\end{array}\right)}}{1-\frac{x y^{\left(\begin{array}{c}
k+1 \\
2
\end{array}\right)}}{1-\frac{x y^{\left(\begin{array}{c}
k+2 \\
3
\end{array}\right)}}{\ldots}}}}
$$

is denoted by $S_{j}(x, y ; k)$, or just $S_{j}$ when the value of $k$ is clear from the context. Our interest in (2) is stipulated by the following relation, which is an easy consequence of Theorem 2.1:

$$
F(x, y ; k)=S_{k}(x, y ; k) .
$$

First of all, we find an explicit formula for the functions $R_{j}(x)$. Let $U_{j}(\cos \theta)=$ $\sin (j+1) \theta / \sin \theta$ be the Chebyshev polynomials of the second kind.

Lemma 3.1. For any $j \geqslant 1$

$$
R_{j}(x)=\frac{U_{j-1}\left(\frac{1}{2 \sqrt{x}}\right)}{\sqrt{x} U_{j}\left(\frac{1}{2 \sqrt{x}}\right)} .
$$

Proof. Indeed, it follows immediately from $(2)$ that $R_{j}(x)$ is the $j$ th approximant for the continued fraction

$$
\frac{1}{1-\frac{x}{1-\frac{x}{1-\frac{x}{\cdots}}}}
$$


Hence, by [7, Theorem 2, p. 194], for any $j \geqslant 1$ one has $R_{j}(x)=A_{j}(x) / A_{j+1}(x)$, where

$$
A_{j}(x)=\left(\frac{1+\sqrt{1-4 x}}{2}\right)^{j}-\left(\frac{1-\sqrt{1-4 x}}{2}\right)^{j} .
$$

Using substitution $x \rightarrow 1 / 4 t^{2}$ one gets $(2 t)^{j} A_{j}\left(1 / 4 t^{2}\right)=2 \sqrt{t^{2}-1} U_{j-1}(t)$, which gives $A_{j}(x)=\sqrt{1 / x-4} x^{j / 2} U_{j-1}(1 / 2 \sqrt{x})$, and the result follows.

Next, we find an explicit expression for $S_{j}$ in terms of $G$ and $R_{j}$.

Lemma 3.2. For any $j \geqslant 1$ and any $k \geqslant 1$

$$
S_{j}(x, y ; k)=R_{j}(x) \frac{1-x R_{j-1}(x) G(x, y ; k)}{1-x R_{j}(x) G(x, y ; k)} .
$$

Proof. Indeed, from (2) and $S_{0}=G$ we get $S_{1}=1 /(1-x G)$. On the other hand, $R_{0}=0, R_{1}=1$, so (5) holds for $j=1$. Now let $j>1$, then by induction

$$
S_{j}=\frac{1}{1-x S_{j-1}}=\frac{1}{1-x R_{j-1}} \cdot \frac{1-x R_{j-1} G}{1-\frac{x\left(1-x R_{j-2}\right) R_{j-1} G}{1-x R_{j-1}}} .
$$

Relation (2) for $R_{j}$ and $R_{j-1}$ yields $\left(1-x R_{j-2}\right) R_{j-1}=\left(1-x R_{j-1}\right) R_{j}=1$, which together with the above formula gives (5).

As a corollary from Lemma 3.2 and (3) we get the following expression for the generating function $F(x, y ; k)$.

\section{Corollary.}

$$
F(x, y ; k)=R_{k}(x)+\left(R_{k}(x)-R_{k-1}(x)\right) \sum_{m \geqslant 1}\left(x R_{k}(x) G(x, y ; k)\right)^{m} .
$$

Now we are ready to express the generating functions $F_{r}(x ; k), 0 \leqslant r \leqslant k$, via Chebyshev polynomials.

Theorem 3.1. For any $k \geqslant 1, F_{r}(x ; k)$ is a rational function given by

$$
\begin{aligned}
& F_{r}(x ; k)=\frac{x^{\frac{r-1}{2}} U_{k-1}^{r-1}\left(\frac{1}{2 \sqrt{x}}\right)}{U_{k}^{r+1}\left(\frac{1}{2 \sqrt{x}}\right)}, \quad 1 \leqslant r \leqslant k, \\
& F_{0}(x ; k)=\frac{U_{k-1}\left(\frac{1}{2 \sqrt{x}}\right)}{\sqrt{x} U_{k}\left(\frac{1}{2 \sqrt{x}}\right)},
\end{aligned}
$$


where $U_{j}$ is the $j$ th Chebyshev polynomial of the second kind.

Proof. Observe that $G(x, y ; k)=y+y^{k+1} P(x, y)$, so from Corollary we get

$$
F(x, y ; k)=R_{k}(x)+\left(R_{k}(x)-R_{k-1}(x)\right) \sum_{m=1}^{k}\left(x R_{k}(x)\right)^{m} y^{m}+y^{k+1} P^{\prime}(x, y),
$$

where $P(x, y)$ and $P^{\prime}(x, y)$ are formal power series. To complete the proof, it suffices to use (4) together with the identity $U_{n-1}^{2}(z)-U_{n}(z) U_{n-2}(z)=1$, which follows easily from the trigonometric identity $\sin ^{2} n \theta-\sin ^{2} \theta=\sin (n+1) \theta \sin (n-1) \theta$.

For the case $r=0$ this result was proved by a different method in [4].

\section{Further Results}

There are several ways to generalize the results of the previous sections. First, one can try to get exact formulas for $F_{r}(x ; k)$ in the case $r>k$. The method described in Section 3 allows, in principle, to obtain such formulas, though they become more and more complicated. For example, the following theorem gives an explicit expression for $F_{r}(x ; k)$ when $r \leqslant k(k+3) / 2$.

Theorem 4.1. For any $k \geqslant 1$ and $1 \leqslant r \leqslant k(k+3) / 2, F_{r}(x ; k)$ is a rational function given by

$$
F_{r}(x ; k)=\frac{x^{\frac{r-1}{2}} U_{k-1}^{r-1}\left(\frac{1}{2 \sqrt{x}}\right)}{U_{k}^{r+1}\left(\frac{1}{2 \sqrt{x}}\right)} \sum_{j=0}^{\lfloor(r-1) / k\rfloor}\left(\begin{array}{c}
r-k j+j-1 \\
j
\end{array}\right)\left(\frac{U_{k}\left(\frac{1}{2 \sqrt{x}}\right)}{x^{\frac{k-2}{2 k}} U_{k-1}\left(\frac{1}{2 \sqrt{x}}\right)}\right)^{k j},
$$

where $U_{j}$ is the $j$ th Chebyshev polynomial of the second kind.

Proof. Indeed, the explicit expression for $G(x, y ; k)$ gives

$$
G(x, y ; k)=y\left(1+x y^{k}+\cdots+x^{s} y^{k s}\right)+y^{t} P(x, y),
$$

where $s=\lceil(k+1) / 2\rceil, t=1+k(k+3) / 2$, and $P(x, y)$ is a formal power series. Hence, by Corollary,

$$
\begin{aligned}
\frac{F(x, y ; k)-R_{k}(x)}{R_{k}(x)-R_{k-1}(x)} & =\sum_{m \geqslant 1}\left(x R_{k}(x)\right)^{m} y^{m}\left(1+x y^{k}+\cdots+x^{s} y^{k s}\right)^{m}+y^{t} P^{\prime}(x, y) \\
& =\sum_{m \geqslant 1}\left(x R_{k}(x)\right)^{m} y^{m} \sum_{j=0}^{m s}\left(\begin{array}{c}
m+j-1 \\
j
\end{array}\right) x^{j} y^{k j}+y^{t} P^{\prime}(x, y) \\
& =\sum_{r \geqslant 1} y^{r}\left(x R_{k}(x)\right)^{r} \sum_{j=0}^{\lfloor(r-1) / k\rfloor} \frac{\left(\begin{array}{c}
r-k j+j-1 \\
j
\end{array}\right) x^{j}}{\left(x R_{k}(x)\right)^{k j}}+y^{t} P^{\prime \prime}(x, y),
\end{aligned}
$$


where $P^{\prime}(x, y)$ and $P^{\prime \prime}(x, y)$ are formal power series. The rest of the proof follows the proof of Theorem 3.1.

Another possibility is to analyze the case of permutations containing exactly one 132 pattern and $r 12 \ldots k$ patterns. Introducing the modified total weight $\Omega_{k}\left(q_{1}, \ldots, q_{k}\right)$ as the sum of the weights $w_{k}(\pi)$ over all permutations containing exactly one 132 pattern, we get the following equation:

$$
\begin{aligned}
\Omega_{k}\left(q_{1}, \ldots, q_{k}\right) & =q_{1} W_{k}\left(q_{1} q_{2}, \ldots, q_{k-1} q_{k}, q_{k}\right) \Omega_{k}\left(q_{1}, \ldots, q_{k}\right) \\
& +q_{1} W_{k}\left(q_{1}, \ldots, q_{k}\right) \Omega_{k}\left(q_{1} q_{2}, \ldots, q_{k-1} q_{k}, q_{k}\right) \\
& +q_{1}^{2} q_{2}^{2} W_{k}\left(q_{1} q_{2}, \ldots, q_{k-1} q_{k}, q_{k}\right)\left(W_{k}\left(q_{1}, \ldots, q_{k}\right)-1\right)
\end{aligned}
$$

for the case $k=3$ see [10]. By (1) and Proposition 2.3 this is equivalent to

$$
\begin{aligned}
\Omega_{k}\left(\mathbf{q}^{d, 1}, \ldots, \mathbf{q}^{d, k}\right) & =\mathbf{q}^{d, 1}\left(\mathbf{q}^{d, 2}\right)^{2}\left(W_{k}\left(\mathbf{q}^{d, 1}, \ldots, \mathbf{q}^{d, k}\right)-1\right)^{2} \\
& +\mathbf{q}^{d, 1} W_{k}^{2}\left(\mathbf{q}^{d, 1}, \ldots, \mathbf{q}^{d, k}\right) \Omega_{k}\left(\mathbf{q}^{d+1,1}, \ldots, \mathbf{q}^{d+1, k}\right) .
\end{aligned}
$$

Let now $\varphi_{n}^{r}(k)$ be the number of permutations on $n$ letters that contain exactly one 132 pattern and $r 12 \ldots k$ patterns, and $\Phi_{r}(x ; k)$ be the generating function of the sequence $\left\{\varphi_{n}^{r}(k)\right\}$ for a given $r$. In general, equation (6) allows us to find explicit expressions for $\Phi_{r}(x ; k)$. However, they are rather cumbersome, so we restrict ourselves to the case $r=0$.

Theorem 4.2. For any $k \geqslant 3, \Phi_{0}(x ; k)$ is a rational function given by

$$
\begin{aligned}
\Phi_{0}(x ; k) & =\frac{x}{U_{k}^{2}\left(\frac{1}{2 \sqrt{x}}\right)} \sum_{j=1}^{k-2} U_{j}^{2}\left(\frac{1}{2 \sqrt{x}}\right) \\
& =\frac{1}{16 \sin ^{2}(k+1) t \cos ^{2} t}\left(2 k-5+4 \cos ^{2} t-\frac{\sin (2 k-1) t}{\sin t}\right),
\end{aligned}
$$

where $U_{j}$ is the $j$ th Chebyshev polynomial of the second kind and $\cos t=1 / 2 \sqrt{x}$. 


\section{REFERENCES}

1. M. Bona, Permutations avoiding certain patterns: the case of length 4 and some generalizations, Discr. Math. 175 (1997), 55-67.

2. M. Bona, The number of permutations with exactly $r$ 132-subsequences is $P$-recursive in the size!, Adv. Appl. Math. 18 (1997), 510-522.

3. M. Bona, Permutations with one or two 132-subsequences, Discr. Math. 181 (1998), 267-274.

4. T. Chow and J. West, Forbidden subsequences and Chebyshev polynomials, Discr. Math. 204 (1999), 119-128.

5. D. Knuth, The Art of Computer Programming, vol. 3, Addison Wesley, Reading, MA, 1973.

6. V. Lakshmibai and B. Sandhya, Criterion for smoothness of Schubert varieties in $\mathrm{Sl}(n) / B$, Proc. Indian Acad. Sci. 100 (1990), no. 1, 45-52.

7. L. Lorentzen and H. Waadeland, Continued fractions with applications, North-Holland, 1992.

8. J. Noonan, The number of permutations containing exactly one increasing subsequence of length three, Discr. Math. 152 (1996), 307-313.

9. J. Noonan and D. Zeilberger, The enumeration of permutations with a prescribed number of "forbidden" patterns, Adv. Appl. Math. 17 (1996), 381-407.

10. A. Robertson, H. Wilf, and D. Zeilberger, Permutation patterns and continuous fractions, Elec. J. Comb. 6 (1999), no. 1, R38.

11. R. Simion and F. Schmidt, Restricted permutations, Europ. J. Comb. 6 (1985), 383-406.

12. Z. Stankova, Classification of forbidden subsequences of length 4, Eur. J. Comb. 17 (1996), 501-517.

13. Z. Stankova, Forbidden subsequences, Discr. Math. 132 (1994), 291-316.

14. J. West, Generating trees and the Catalan and Schröder numbers, Discr. Math. 146 (1995), $247-262$. 